\title{
Higher Rightward Laterality of the Hippocampal Tail and Its Association with Early Trauma in Panic Disorder
}

\author{
Joonho Lee', Yoonsu Song ${ }^{1}$, Eunsoo Won ${ }^{1}$, Minji Bang ${ }^{1}$, Sang-Hyuk Lee ${ }^{1,2}$ \\ Departments of ${ }^{1}$ Psychiatry, ${ }^{2}$ Clinical Pharmacology and Therapeutics, CHA Bundang Medical Center, CHA University School of Medicine, \\ Seongnam, Korea
}

\begin{abstract}
Objective: Early trauma (ET) is widely recognized as a contributing factor to the development of panic disorder (PD) in patients. However, there is a dearth of research on the specific volumes of hippocampal subregions and their laterality with respect to ET and PD.

Methods: A total of 30 subjects with PD and 30 age- and sex-matched healthy controls (HCs) were included in this study. All the subjects were evaluated by 3T-magnetic resonance imaging. FreeSurfer version 6.0 was used for volumetric analysis of the hippocampal subregions and their laterality. A shortened version of the Early Trauma Inventory Self Report (ETISR) as well as Anxiety Sensitivity Inventory-Revised (ASI-R), and Panic Disorder Severity Scale were utilized for analysis.

Results: Multivariate analysis of variance showed that the volume of the right hippocampal tail and laterality indices (LIs) of the hippocampal body and tail were significantly larger in subjects with PD relative to HCs. The significance of the observations remained unchanged after multivariate analysis of covariance, controlling for age, sex, years of education, medication, depressive symptoms, and intracranial volume as covariates. The Lls of the hippocampal tails that showed a significant correlation to ETISR emotional and physical subscales were also associated with ASI-R for cardiovascular symptoms in PD.

Conclusion: Our study displayed an increased rightward lateralization of the hippocampal tails in subjects with PD compared with HCs. This alteration in the brain, which was associated with early emotional and physical trauma, would negatively affect anxiety sensitivity to cardiovascular symptoms in subjects with PD.
\end{abstract}

KEY WORDS: Panic disorder; Hippocampus; Psychological trauma; Vulnerability; Gray matter; Magnetic resonance imaging.

\section{INTRODUCTION}

Panic disorder (PD) is characterized by recurrent panic attacks and anticipatory anxiety, concomitant multiple somatic symptoms (e.g., palpitations, sweating, shaking, shortness of breath, and numbness) and/or a feeling that

Received: September 24, 2019 / Revised: December 23, 2019

Accepted: February 17, 2020

Address for correspondence: Sang-Hyuk Lee

Departments of Psychiatry, Clinical Pharmacology and Therapeutics, CHA Bundang Medical Center, CHA University School of Medicine, 59 Yatap-ro, Bundang-gu, Seongnam 13496, Korea

E-mail: leesanghyuk@yahoo.com

ORCID: https://orcid.org/0000-0001-7939-3000

Minji Bang

Department of Psychiatry, CHA Bundang Medical Center, CHA

University School of Medicine, 59 Yatap-ro, Bundang-gu, Seongnam

13496, Korea

E-mail: minjibang@cha.ac.kr

ORCID: https://orcid.org/0000-0002-1669-4014 something terrible is going to happen. Due to the high recurrence and debilitating nature of panic symptoms, PD is often considered by clinicians as a chronic condition [1]

Available reports in the field have consistently reported the etiology of PD as multifactorial, including early traumatic life events as a contributing factor [2]. Children who have experienced trauma become increasingly responsive to minor stimuli due to the sensitization and kindling of the fear system [3]. In addition, the dysfunction of fear network has been known to be closely associated with early childhood life stressors [4]. Since PD is a disorder of the fear network $[4,5]$, aberrant structural and functional changes in the brain regions involved in this network would be implicated in the pathogenesis of PD. Neuroimaging studies have suggested that the hippocampus, the essential brain structure for fear conditioning within the fear circuit [6], is one of the most critical brain regions

(ㄷ) This is an Open-Access article distributed under the terms of the Creative Commons Attribution Non-Commercial License (http://creativecommons.org/licenses/by-nc/4.0) which permits unrestricted non-commercial use, distribution, and reproduction in any medium, provided the original work is properly cited. 
which is vulnerable to traumatic life experiences [7-10]. This region plays a central role in contextual fear conditioning $[11,12]$, which leads to anxiety responses in the context in which threat was experienced [13]. Heightened contextual fear conditioning and its generalization confer susceptibility to anxiety and panic symptoms and contribute to the characteristic avoidance of anxiety contexts and withdrawal to safety contexts in patients with PD [14]. A meta-analysis [15] reported that trauma-exposed adults showed volume deficits in the hippocampus, independent of the diagnosis of post-traumatic stress disorder, thereby suggesting that the hippocampus is adversely affected by traumatic life experiences. However, such volumetric aberrations of the hippocampus were not observed in studies on children and adolescents [16]. This inconsistency implies that the timing of trauma exposure is associated with distinct neurobiological effects on the hippocampus.

From the neurodevelopmental perspective, the hippocampus is known to develop rapidly during the early postnatal years [17], followed by steady growth and maturation until adolescence [18]. Therefore, this region would be one of the most vulnerable areas to the adverse effects of trauma during childhood and adolescence. A longitudinal study examining normal hippocampal development in children and adolescents revealed distinct developmental trajectories of hippocampal subregions along the longitudinal axis. The posterior part of the hippocampus showed gradual gain over time, more prominently on the left; while the anterior part displayed reduction in volume over time [19]. Additionally, previous neuroimaging studies $[20,21]$ have shown that when retrieving specific episodic memories, children recruit the posterior part of the hippocampus rather than the anterior part, which is more involved among adults [21]. Therefore, it could be assumed that the posterior hippocampus, the development of which predominantly occurs during childhood, might be particularly affected by early life trauma [22]. Although, there have been few attempts to investigate the volumetric aberrations of the hippocampus during PD, the results have been rather inconsistent. Vythilingam et al. [23] argued in a preliminary study that there was no significant difference in the hippocampal volume between patients with PD and healthy controls, whereas Dantendorfer et al. [24] observed smaller hippocampal volumes in some PD patients. These inconsistencies could be partly attributed to the distinct developmental patterns in the left and right subregions of the hippocampus, suggesting that early traumatic life events might not only alter the absolute volumes of the hippocampal subregions; but also modify the normative lateralization in the hippocampal subregions.

Albeit widely recognized, hemispheric asymmetry of the hippocampus has been attracting attention only recently. In fact, the two hippocampi are both anatomically and functionally distinct. The right hippocampus is normally larger, and has a specific role in spatial memory, whereas the left hippocampus mediates verbal memory $[25,26]$. Such functional and structural distinctions between the two hippocampi shed light on the importance of hippocampal laterality in psychiatric disorders. An magnetic resonance imaging (MRI) study of healthy individuals revealed that right-sided $(\mathrm{R}>\mathrm{L}$ ) anterior hippocampal volume asymmetry predicts basic cognitive abilities [27]. High scores on the Sensitivity to Punishment scale were positively associated with hippocampal volume, and this phenomenon was lateralized to the right side [28]. A comparative study among individuals with schizophrenia, their siblings, and healthy controls (HCs) revealed that the normative pattern of right-sided hippocampal asymmetry was aberrantly altered in patients with schizophrenia and their siblings [29]. On similar lines, relevant hippocampal morphometric [30] and glutamatergic [31] data indicate asymmetric neuropathological involvement of the hippocampus in schizophrenia [26].

The objective of this study is to explore hippocampal subregions and their laterality in order to identify a possible explanatory linkage between early traumatic events, such as physical and emotional abuse, and their contribution to alterations in hippocampal subregions and laterality, which may be associated with the development of trait or state symptoms of PD. We hypothesized the following: first, there could be alterations in the volume of the hippocampus, including its subregions and their laterality in patients with PD compared to HCs. Secondly, there could be a significant association between alterations in volumes and laterality of hippocampal subregions and early trauma (ET). Furthermore, there could be a correlation between the structural hippocampal alterations associated with ET and trait or state markers of PD. 


\section{METHODS}

\section{Subjects and Clinical Assessment}

The subjects in this study were recruited from patients who visited the Department of Psychiatry of CHA Bundang Medical Center between January 2011 and August 2019 and were diagnosed with PD. The HCs were recruited through advertisements. A total of 30 subjects with PD and 30 age- and sex-matched HCs were included in this study. All subjects were Korean right-handed individuals between 18 and 65 years of age. Hand preference of the subjects was assessed by the Edinburgh Handedness Inventory measurement scale [32]. All HCs were interviewed to ensure that they had no personal or family history of psychiatric disorders among first-degree relatives. Diagnoses of PD with or without agoraphobia were established by experienced psychiatrists through the Structured Clinical Interview for Diagnostic and Statistical Manual of Mental Disorders, 4th Edition, Text Revision (DSM-IV-TR) Axis I Disorders [33]. The patients with only primary diagnoses of PD were included in this study. No subject with PD met the criteria for other Axis I disorders. The subjects who reported of any concurrent diagnosis or lifetime history of schizophrenia, bipolar disorder, depressive disorder, anxiety disorders other than PD, substance abuse or dependence, mental retardation, serious medical disorders, pregnancy, or contraindications to brain magnetic resonance (MR) scanning were excluded from the study. In the subjects, the mean age of the onset of PD was 33.4 years and the mean duration of illness was 64.9 months before the study initiation. Sixteen subjects with PD who were on antidepressants for a brief period, discontinued their medication before MRI scan evaluation. The brain MRI was performed $18.13 \pm 37.91$ days after the initiation of the use of medication. After the MRI scan, all subjects with PD, including 14 antidepressant-naïve ones, were medicated with a minimal dosage of selective serotonin reuptake inhibitors and serotonin and norepinephrine reuptake inhibitors. These included escitalopram, paroxetine, fluoxetine, and duloxetine ( $\mathrm{n}=30$; escitalopram equivalence dosage, $9.67 \pm 13.32 \mathrm{mg} /$ day), and benzodiazepines such as alprazolam, clonazepam, lorazepam and flurazepam ( $\mathrm{n}=30$; alprazolam equivalence dosage, $0.90 \pm 0.90 \mathrm{mg} /$ day) .

A shortened version of the Early Trauma Inventory Self Report (ETISR-SF) has been developed and is now avail- able for the evaluation of ET [34]. This study adopted the Korean version of ETISR-SF [35], which consists of 27 yes-no questions assessing the four domains of physical, emotional, sexual, and general trauma experienced before the age of 18 years.

To evaluate the potential trait markers of PD, the Korean version of Anxiety Sensitivity Inventory-Revised (ASI-R) [36,37] was used to assess the anxiety sensitivity (AS) levels of all subjects. The internal consistency coefficient of the Korean version is 0.92 and its test-retest reliability is 0.82 [36]. ASI-R, the most commonly used measure for AS, comprises the fear of respiratory symptom, fear of cardiovascular symptom, fear of publicly observable anxiety reaction, and fear of cognitive dyscontrol. Each item is scored on a scale from 0 (very little) to 4 (very much) and the total score ranges from 0 to 144 .

Further, in order to assess the potential state markers of PD, the severity of panic and depressive symptoms was assessed in each subject using the Panic Disorder Severity Scale $[38,39]$. To measure the clinical severity of subjects' anxiety and depressive symptoms, we concurrently applied the Albany Panic and Phobia Questionnaire [40], the Beck Anxiety Inventory [41], and the Beck Depression Inventory-II (BDI-II) [42]. The scores of all the three scales were determined within 3 days before or after the MRI. The Scale for Suicide Ideation [43], which gives a high predictive value for suicide [44], was also used to measure the seriousness of suicidal ideation in each subject.

This study was approved by the CHA Bundang Medical Center Ethics Committee. All study procedures complied with the CHA Bundang Medical Center Institutional Review Board regulations (no. 2011-11-164, 2018-06-029, 2019-05-030), the Declaration of Helsinki, and the Principles of Good Clinical Practice. It was ascertained that all subjects had fully understood the research protocol and their verbal and written consents were obtained after they had thoroughly reviewed a full description of the study.

\section{MRI Acquisition and Hippocampal Segmentations}

3T GE Signa HDxt scanner (GE Healthcare, Milwaukee, WI, USA) with an eight-channel phase array head coil was used to perform MRI of the brain in all subjects at the $\mathrm{CHA}$ Bundang Medical Center, CHA University. The following parameters were used for three-dimensional T1-weighted fast spoiled gradient recalled echo (T1-FSPGR) image ac- 
quisition: repetition time, $6.3 \mathrm{~ms}$; echo time, $2.1 \mathrm{~ms}$; flip angle, $12^{\circ}$; slice thickness, $1 \mathrm{~mm}$; field of view, $25.6 \mathrm{~cm}$; $256 \times 256$ matrix; isotropic voxel size, $1 \times 1 \times 1 \mathrm{~mm}^{3}$. FreeSurfer version 6.0.0 was utilized to process the MR images and automatically segment the hippocampus into distinct subregions. The image processing included the following steps: averaging of the volumetric T1-FSPGRweighted images, removal of non-brain tissue and Talairach transformation, volumetric segmentation, intensity normalization, tessellation of the gray matter (GM)-white matter (WM) boundary, topology correction, and surface deformation following intensity gradients. The details of the procedure are available in the previous research articles $[45,46]$.

The algorithm of the hippocampal subfield segmentation in FreeSurfer, validated using the ADNI dataset, was established by examining the data from 15 autopsy scans of $0.13 \mathrm{~mm}$ isotropic resolution using Bayesian inference methodology [46]. The segmentation processes used statistical inference to identify subregions of interest. The segmentation process utilized both T1- and T2-weighted images for maximized accuracy. J.L. visually inspected all MR images to avoid any reconstruction errors, such as skull-stripping errors, gross parcellation errors, and WM and pial surface inaccuracies. Surface inaccuracies were also manually corrected using FreeSurfer's editing protocols in accordance with an internal, standardized quality control. To improve the accuracy of the automatic tracing method, the FreeSurfer pipeline was used to reprocess the edited images and visual inspection was done for all output images. The hippocampus was segmented into 19 subfields, that were regrouped into (1) the head (including parasubiculum, presubiculum-head, subiculum-head, CA1head, CA3-head, CA4-head, GC-ML-DG-head, molec-
ular_layer_HP-head, and HATA), (2) body (including presubiculum-body, subiculum-body, CA1-body, CA3-body, CA4-body, GC-ML-DG-body, molecular_layer_HP-body, and fimbria), (3) tail and, (4) fissure. We selected 3 regions of interest (ROIs) in both the left and the right hippocampi: the head, body, and tail of the hippocampus (Fig. 1) [46].

We calculated the laterality index (LI) [47] for each ROI with a standard formula as follows:

$$
\mathrm{LI}=\frac{V_{R}-V_{L}}{V_{R}+V_{L}} \times 2
$$

where $\mathrm{LI}$ is the laterality index, $V_{R}$ is the volume of the hippocampal subregion on the right hemisphere, and $V_{L}$ the volume of the hippocampal subregion on the left hemisphere.

\section{Statistical Analysis}

Independent $t$ test and chi-square $\left(\chi^{2}\right)$ tests were used to compare sociodemographic and clinical data between subjects with PD and HCs.

Since there were significant correlations with moderate effect sizes in ROls, multivariate analysis of variance (MANOVA) was applied to compare absolute volumes and LIs of each ROI between subjects with PD and HCs. To ensure that the results were not confounded by demographic differences, multivariate analysis of covariance (MANCOVA) was also performed, using age, sex, years of education, intracranial volume (ICV), BDI-II, duration of medication, and medication dosage as covariates. Posthoc multiple comparisons between ROls were performed for both MANOVA and MANCOVA using a Bonferroni method.

Exploratory correlation analyses were applied to eval-
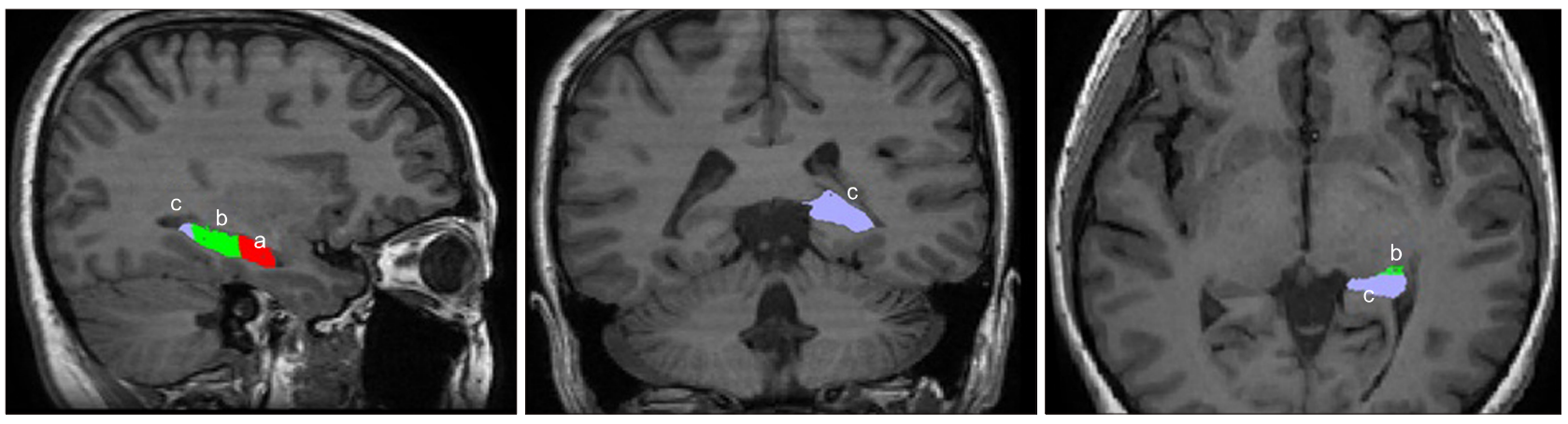

Fig. 1. Regions of Interest of the left hippocampus: (a) head, (b) body, and (c) tail. Orientation is in accordance with radiological convention. 
uate the relationship between ET, trait and state markers of PD and the volumes of the hippocampal subregions and their LIs using Pearson correlation coefficient. Fisher's r-to-z transformation was performed to compare the correlation coefficients between subjects with PD and HCs.

All statistical analyses were performed using IBM SPSS Statistics 23.0 software (IBM Co., Armonk, NY, USA) tools. For all tests, $p<0.05$ was considered as statistically significant.

\section{RESULTS}

\section{Sociodemographic and Clinical Characteristics}

The sociodemographic and clinical characteristics of all study subjects are summarized in Table 1. There was no significant difference between subjects with PD and HCs with respect to sex, age, and ICV. HCs had higher education levels than subjects with PD. All clinical scores were significantly higher in subjects with PD than in HCs. There was no significant correlation between the chronicity of illness and the score for clinical symptoms in any scale. Besides, the chronicity of illness was not shown to be significantly correlated with ICV and hippocampal volumes.

\section{Comparison of Volumes and Laterality Indices of Hippocampal Subregions between Subjects with Panic Disorder and Healthy Controls}

A significant difference in the $\mathrm{LI}$ of the whole hippocampal volume was found between subjects with PD and HCs $(t=3.13, p=0.003)$, but not the total hippocampal volume (left hippocampus; $t=-0.11, p=0.910$, right hippocampus; $t=1.64, p=0.106)$. There was no significant association between the $\mathrm{LI}$ of the whole hippocampal volume and any clinical scores in the scales of PD.

MANOVA (Table 2) revealed significant between-group

Table 1. Demographic and clinical characteristics of study subjects

\begin{tabular}{|c|c|c|c|c|}
\hline Variable & $\mathrm{PD}(\mathrm{n}=30)$ & HCs $(n=30)$ & Statistics & $p$ value \\
\hline \multicolumn{5}{|l|}{ Sex } \\
\hline Male & $18(60.0)$ & $18(60.0)$ & $\chi^{2}<0.001$ & 1.000 \\
\hline Female & $12(40.0)$ & $12(40.0)$ & & \\
\hline Age (yr) & $39.6 \pm 13.4$ & $41.8 \pm 12.5$ & $t=-0.66$ & 0.512 \\
\hline Intracranial volume (ml) & $1,510.0 \pm 126.5$ & $1,526.5 \pm 132.8$ & $t=-0.48$ & 0.631 \\
\hline Years of education (yr) & $14.1 \pm 2.2$ & $16.0 \pm 0.9$ & $t=-4.40$ & $<0.001$ \\
\hline Duration of illness (mo) & $64.9 \pm 104.3$ & & & \\
\hline Agoraphobia & $12(40.0)$ & & & \\
\hline Family history of anxiety disorder & $6(20.0)$ & $0(0)$ & & $0.305^{\mathrm{a}}$ \\
\hline Baseline ETISR-SF & $5.3 \pm 4.4$ & $3.0 \pm 3.5$ & $t=1.47$ & 0.150 \\
\hline General trauma & $1.6 \pm 2.0$ & $0.7 \pm 0.8$ & $t=1.40$ & 0.169 \\
\hline Physical trauma & $2.0 \pm 1.8$ & $1.5 \pm 1.9$ & $t=0.76$ & 0.454 \\
\hline Emotional trauma & $1.5 \pm 1.8$ & $0.7 \pm 1.6$ & $t=1.22$ & 0.232 \\
\hline Sexual trauma & $0.2 \pm 0.6$ & $0.1 \pm 0.3$ & $t=0.49$ & 0.624 \\
\hline Baseline ASI-R total score & $44.1 \pm 23.4$ & $2.3 \pm 3.6$ & $t=9.55$ & $<0.001$ \\
\hline Fear of respiratory symptom & $16.5 \pm 10.8$ & $0.3 \pm 0.6$ & $t=8.21$ & $<0.001$ \\
\hline Fear of cardiovascular symptom & $10.5 \pm 8.6$ & $0.9 \pm 2.2$ & $t=5.72$ & $<0.001$ \\
\hline Fear of publicly observable anxiety reaction & $11.2 \pm 8.0$ & $1.1 \pm 2.1$ & $t=6.47$ & $<0.001$ \\
\hline Fear of cognitive dyscontrol & $5.8 \pm 5.9$ & $0.0 \pm 0.0$ & $t=5.44$ & $<0.001$ \\
\hline Baseline APPQ total score & $49.1 \pm 36.9$ & $9.1 \pm 6.9$ & $t=5.71$ & $<0.001$ \\
\hline Agoraphobia & $18.2 \pm 15.8$ & $2.6 \pm 2.0$ & $t=5.34$ & $<0.001$ \\
\hline Social phobia & $17.0 \pm 15.5$ & $4.3 \pm 4.2$ & $t=4.18$ & $<0.001$ \\
\hline Interoceptive fear & $13.8 \pm 12.2$ & $2.3 \pm 2.2$ & $t=5.02$ & $<0.001$ \\
\hline Baseline BAI total score & $23.8 \pm 15.8$ & $0.9 \pm 1.6$ & $t=7.83$ & $<0.001$ \\
\hline Baseline BDI-II total score & $16.6 \pm 10.3$ & $2.3 \pm 2.4$ & $t=7.19$ & $<0.001$ \\
\hline Baseline SSI total score & $4.8 \pm 7.6$ & $0.2 \pm 0.4$ & $t=3.29$ & 0.003 \\
\hline
\end{tabular}

Values are presented as number $(\%)$ or mean \pm standard deviation.

PD, panic disorder; HCs, healthy controls; ETISR-SF, Early Trauma Inventory Self Report-Short Form; ASI-R, Anxiety Sensitivity Inventory-Revised; APPQ, Albany Panic and Phobia Questionnaire; BAI, Beck Anxiety Inventory; BDI-II, Beck Depression Inventory-II; SSI, Scale for Suicidal Ideation. 'Fisher's exact $p$. 
differences in the volumes of the right hippocampal ROIs, but not in the left. Post-hoc analysis revealed that the volume of the right hippocampal tail was significantly larger in subjects with PD relative to HCs after Bonferroni correction for multiple comparisons, while the other hippocampal ROls did not show any significant difference. However, there was no significant association between the volume of the right hippocampal tail and any clinical scores in scales of PD.

MANOVA (Table 3) revealed a significant between-group difference in the LI of hippocampal ROls. Post-hoc analysis revealed that the laterality of the hippocampal body and tail was significantly larger in subjects with PD relative to HCs after Bonferroni correction for multiple comparisons. There was no significant difference in the LI of the hippocampal head between subjects with PD and HCs.

The significance of the results remained the same after MANCOVA using age, sex, years of education, ICV, BDI-II, duration of medication, and medication dosage as covariates.

\section{Correlation between Laterality Indices of Hippocampal Subregions and Early Trauma History in Subjects with Panic Disorder and Healthy Controls}

Exploratory correlation analysis using Pearson correlation was performed to evaluate the relationship between the LIs of hippocampal ROIs and early trauma inventory scores. There were significant correlations between the LI of the hippocampal tail and ETISR physical $(r=0.367, p=$ $0.046)$ and emotional scores $(r=0.505, p=0.004$; Fig. $2 \mathrm{~A}, 2 \mathrm{~B})$. Thus, greater the physical or emotional trauma of a patient, higher was the rightward $\mathrm{LI}$ of his/her hippocampal tail. The significant result remained grossly unchanged for ETISR emotional scores after partial correlation analysis using age, sex, years of education, ICV, $\mathrm{BDI}-\mathrm{II}$, duration of medication, and medication dosage as covariates, but not for ETISR physical scores.

In $\mathrm{HCs}$, on the contrary, no significant correlation between the $\mathrm{LI}$ of the hippocampal subregions and ETISR subscores was found. When the significance of the difference between the correlation coefficients of subjects with $\mathrm{PD}$ and HCs was examined using the Fisher's r-to-z transformation, only the correlation coefficient of the ETISR

Table 2. Multivariate analysis of variance on volumes of the hippocampal ROls between subjects with PD and HCs

\begin{tabular}{cccccc}
\hline Hippocampal volume $\left(\mathrm{mm}^{3}\right)$ & PD $(\mathrm{n}=30)$ & HCs $(\mathrm{n}=30)$ & $F$ & $p$ value $^{\mathrm{c}}$ & $\mathrm{ES}^{\mathrm{d}}$ \\
\hline Left $^{\mathrm{a}}$ & & & & & \\
Head & $1,762.5 \pm 173.0$ & $1,791.7 \pm 224.3$ & & & \\
Body & $1,286.2 \pm 97.8$ & $1,287.5 \pm 156.1$ & & & \\
Tail & $616.9 \pm 81.2$ & $596.5 \pm 83.4$ & & & \\
Right $^{\mathrm{b}}$ & & & & & \\
Head & $1,868.7 \pm 190.2$ & $1,862.6 \pm 209.3$ & 0.01 & $>0.999$ & 0.001 \\
Body & $1,353.7 \pm 115.3$ & $1,294.4 \pm 124.8$ & 3.65 & 0.183 & 0.059 \\
Tail & $664.0 \pm 71.2$ & $589.7 \pm 63.9$ & 18.14 & $<0.001$ & 0.238 \\
\hline
\end{tabular}

Values are presented as mean \pm standard deviation.

$\mathrm{ROI}$, region of interest; PD, panic disorder; $\mathrm{HCs}$, healthy controls; ES, effect size.

${ }^{a} \mathrm{No}$ significant between-group effect was found in volumes of the left hippocampal ROIs $\left(F=0.52, p=0.674, \eta_{\mathrm{p}}{ }^{2}=0.027\right)$. ${ }^{\mathrm{b}}$ There was a significant

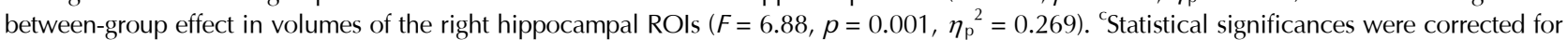
multiple comparisons using a Bonferroni method. ${ }^{\mathrm{d} E S s}$ were calculated using partial eta squared $\left(\eta_{\mathrm{p}}{ }^{2}\right)$.

Table 3. Multivariate analysis of variance on laterality of the hippocampal ROls between subjects with PD and HCs

\begin{tabular}{lccrr}
\hline Laterality index & HCs $(n=30)$ & $F$ & $p$ value $^{\mathrm{b}}$ \\
\hline Head & $0.058 \pm 0.056$ & $0.041 \pm 0.061$ & 1.32 & 0.768 \\
Body & $0.050 \pm 0.054$ & $0.079 \pm 0.072$ & 6.64 & 0.022 \\
Tail & $0.076 \pm 0.094$ & $-0.007 \pm 0.118$ & 9.22 & 0.103 \\
\hline
\end{tabular}

Values are presented as mean \pm standard deviation.

ROI, region of interest; PD, panic disorder; HCs, healthy controls; ES, effect size.

${ }^{\mathrm{a}}$ There was a significant between-group effect in laterality indices of the hippocampal ROls $\left(F=4.43, p=0.007, \eta_{\mathrm{p}}{ }^{2}=0.192\right)$. ${ }^{\mathrm{b}}$ Statistical significances were corrected for multiple comparisons using a Bonferroni method. ${ }^{\mathrm{C}} \mathrm{ESs}$ were calculated using partial eta squared $\left(\eta_{\mathrm{p}}{ }^{2}\right.$ ). 

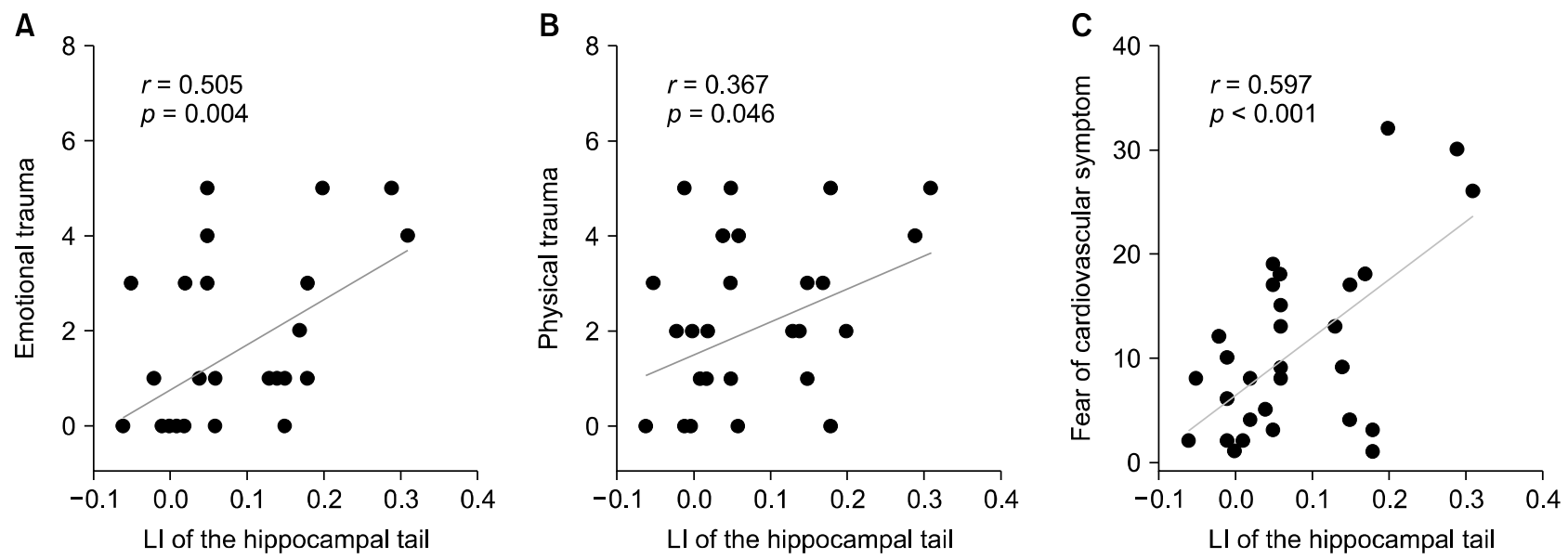

Fig. 2. (A, B) Correlation between laterality index of the hippocampal tail and early emotional and physical trauma in panic disorder. (C) Correlation between laterality index of the hippocampal tail and anxiety sensitivity for cardiovascular symptoms.

$\mathrm{LI}$, laterality index.

emotional scores was significantly different between the two groups $(z=2.05, p=0.040)$.

\section{Correlation between the Laterality Index of the Hippocampal Tail and Trait and State Markers of Panic Disorder}

Pearson correlation analysis was used to evaluate the correlation between the $\mathrm{LI}$ of the hippocampal tail and ASI-R as a trait marker of PD. The LI of the hippocampal tail, which showed significant correlations with physical and emotional trauma, was also significantly correlated with the ASI-R subscale for cardiovascular symptoms ( $r=$ $0.597, p<0.001$; Fig. 2C). There was no significant correlation between the $\mathrm{LI}$ of the hippocampal tail and other clinical scales of PD. The significance of the result remained the same for the ASI-R subscale for cardiovascular symptoms after applying partial correlation using age, sex, years of education, ICV, BDI-II, duration of illness and medication, and medication dosage as covariates.

Conversely, in HCs, no significant correlation between the LI of the hippocampal tail and any clinical scales was found. When the significance of the difference between the correlation coefficients of subjects with PD and HCs was examined using the Fisher's r-to-z transformation, the correlation coefficient of the ASI-R subscale for cardiovascular symptoms was significantly different between the two groups $(z=2.50, p=0.012)$.

\section{DISCUSSION}

To our knowledge, this is the first study that examined aberrant volumetric changes in the hippocampal subregions in $\mathrm{PD}$, and revealed the association between volumes and LIs of hippocampal subregions and ET in PD patients. We also investigated the possible relationship between trait and state markers of PD and the laterality of the hippocampal tail.

In line with our hypothesis that the volumes of hippocampal subregions could be altered in patients with PD, the current study has uncovered significantly increased volumes of the right hippocampal tails in patients with PD when compared with HCs. Acknowledging that our study selected only three hippocampal ROIs along the longitudinal axis, warranting further studies to explore more detailed sections of the hippocampus, the volumetric increase of the right hippocampal tail found in patients with PD could be considered a novel finding. Previous studies have reported volumetric changes in the hippocampus and its subregions in other major psychiatric disorders, such as schizophrenia and depression. The majority of studies have revealed hippocampal volume reduction and altered hippocampal shape in schizophrenia [48,49]. However, a group of researchers have argued that the anterior segment of the hippocampus revealed a pronounced expansion and/or deformation in the right-sided structures ( $\mathrm{R}>\mathrm{L}$ asymmetry) in subjects with schizophrenia compared with HCs [29]. More recently, posterior hippocampus, including the hippocampal tail, has gar- 
nered significant attention in the context of brain imaging studies. This could be largely due to a recent study which involved a large number of depressed patients [50]. Interestingly, this study discovered that exaggerated patterns of hippocampal tail volume disproportion and asymmetry, possibly owing to its high susceptibility to stress-induced vasoconstriction, were associated with the time of remission after antidepressant initiation. Our findings regarding the increased right hippocampal tail volume in subjects with PD might be in concordance with their study. However, since patients with depression were excluded by the exclusion criteria in our study, our novel findings might be implicated for patients with PD, independent of depression as a comorbidity.

Our study found significantly increased rightward LIs in the body and tail of the hippocampus in subjects with PD compared to HCs. This suggests that the alterations in lateralization of the hippocampal body and tail in PD might be related to traumatic memory, fear, and anxiety, since it is well known that the hippocampus plays an important role in the consolidation of information from short-term memory to long-term memory [51] and fear conditioning and affective processes [52]. The LI of the hippocampal tail was positively correlated with physical and emotional ET history in subjects with PD. Essentially, greater is the physical or emotional trauma of a PD patient, higher is the lateralization of the hippocampal tail to the right. Such a significant correlation was not observed in the case of the hippocampal body. This result implies that the hippocampal tail, but not the body, could have a significant role in traumatic memory formation in PD patients. Although there is only limited knowledge on the function of the hippocampal tail, a rodent study suggested that the dorsal hippocampus, which corresponds to the posterior hippocampus in primates, performs primary cognitive functions that correlated with cortical regions involved in information processing [53]. This could explain how the cognitive retrieval of traumatic memories, which is performed by a patient during the assessment of ETISR, could be associated with the lateralization of the hippocampal tail. According to a study that investigated the developmental trajectory of the hippocampus from infancy to early adulthood in healthy individuals, the larger growth rate of the left hippocampus is suspected to be the result of accelerated development in the posterior hippocampal subregion [18]. It has been reported that the differential distribution of $\mathrm{N}$-methyl-D-aspartate receptor subunits between the left and right hippocampus could arouse the lateralization of stress-susceptibility in hippocampus [54]. Since ETISR assesses traumatic memories of childhood and adolescence, it is possible that the increased rightward laterality of hippocampal tail volume in traumatized patients may be attributed to the aberrant lateralizing processes of the hippocampal tail during childhood and adolescence, the essential developmental period of the hippocampus. This result, however, should be interpreted with caution since partial correlation analysis showed a significant correlation between the laterality of the hippocampal tail and emotional trauma, but not physical trauma. This might imply that emotional trauma could be more relevant in the manifestation of the symptomatology of PD than physical trauma. However, more studies would be required to clarify the higher significance of early emotional traumatic experiences than other types of traumas in PD.

Correlation analysis showed that the increased LI of the hippocampal tail is associated with the ASI-R subscale for cardiovascular symptoms in PD. Further, AS for cardiovascular symptoms comprises a large portion of externalized symptoms of panic attacks. Our findings also support our hypothesis that these alterations in hippocampal asymmetry might contribute to sensitization to stress due to the role of the hippocampus in fear generation. This is partly consistent with a previous study that reported that increased stressor-evoked cardiovascular reactivity is associated with altered hippocampal volume [55]. High AS, affected by traumatic experiences during childhood, may sensitize an individual to stressful life events in the adulthood. Furthermore, high AS is known to be associated with an increased tendency of developing PD after stressful life events [56]. Therefore, it could be postulated that the lateralization of the hippocampal tail, influenced by early trauma, would influence AS in the development of PD.

It is noteworthy that, in contrast to subjects with PD, HCs did not show any correlations between the LIs of hippocampal subregions and either ETISR or symptom severity scales of PD. This finding raises the possibility that there might be other factors which play protective roles in the developmental process of the hippocampus. Children with the protective factors might be able to undergo a successful lateralization process of the hippocampus even af- 
ter early traumatic events, with less adverse outcomes in adulthood, such as high AS and development of PD. This interpretation should be considered with caution because we have not conducted investigation of the protective factors in healthy individuals. Also, our findings should be interpreted with a degree of circumscription due to the cross-sectional nature of our study. Longitudinal studies are warranted to determine the causality between early traumatic events and developmental changes in hippocampal laterality.

Several limitations should be considered when interpreting the findings of the present study. First, since we assessed ET in patients with PD retrospectively, the possibility of recall bias cannot be completely ruled out. Secondly, since 16 from a total of 30 subjects with PD were on medication at the time of the MR scan, it is possible that medication may have influenced the hippocampal structure. However, the mean duration of administration of medication (18 days) was relatively short, and the significance of associations remained the same even after correcting for duration of medication administration as a covariate. Thirdly, longitudinal studies are warranted to ensure that the aberrant lateralization of hippocampal subregions is attributable to ET and not a consequential phenomenon of the natural course of PD.

In conclusion, our study shows that the increased lateralization of the hippocampal tail in patients with PD compared to HCs, which may be associated with early trauma, could be correlated with AS for cardiovascular symptoms in PD. Although future studies are necessary, we carefully suggest that our findings might be useful in the investigation of altered asymmetry of hippocampal subregions and could be used as a potential candidate biomarker of AS or symptom severity index in patients with PD.

\section{- Acknowledgments}

This research was supported by the National Research Foundation of Korea (NRF) grants funded by the Ministry of Science and ICT, Republic of Korea (Grant No. NRF2011-0023359, NRF-2018R1D1A1B07046978, and NRF2019M3C7A1032262).

\section{- Conflicts of Interest}

No potential conflict of interest relevant to this article was reported.

\section{Author Contributions}

Conceptualization: Joonho Lee, Sang-Hyuk Lee. Data acquisition: Joonho Lee, Yoonsu Song. Formal analysis: Joonho Lee, Yoonsu Song. Funding: Sang-Hyuk Lee. Supervision: Eunsoo Won, Minji Bang, Sang-Hyuk Lee. Writing-original draft: Joonho Lee, Sang-Hyuk Lee. Writing-review \& editing: Joonho Lee, Minji Bang, Sang-Hyuk Lee.

\section{- ORCID}

Joonho Lee

Yoonsu Song

Eunsoo Won

Minji Bang

Sang-Hyuk Lee

https://orcid.org/0000-0001-9947-1643 https://orcid.org/0000-0002-5908-1349 https://orcid.org/0000-0001-6825-032X https://orcid.org/0000-0002-1669-4014 https://orcid.org/0000-0001-7939-3000

\section{REFERENCES}

1. Liebowitz MR. Panic disorder as a chronic illness. J Clin Psychiatry 1997;58 Suppl 13:5-8.

2. Bandelow B, Krause J, Wedekind D, Broocks A, Hajak G, Rüther E. Early traumatic life events, parental attitudes, family history, and birth risk factors in patients with borderline personality disorder and healthy controls. Psychiatry Res 2005; 134:169-179.

3. Post RM, Weiss SR, Li H, Smith MA, Zhang LX, Xing G, et al. Neural plasticity and emotional memory. Dev Psychopathol 1998;10:829-855.

4. Lai CH. Fear network model in panic disorder: the past and the future. Psychiatry Investig 2019;16:16-26.

5. Goddard AW. The neurobiology of panic: a chronic stress disorder. Chronic Stress 2017;1:1-14.

6. Maren S. Pavlovian fear conditioning as a behavioral assay for hippocampus and amygdala function: cautions and caveats. Eur J Neurosci 2008;28:1661-1666.

7. Tottenham N, Sheridan MA. A review of adversity, the amygdala and the hippocampus: a consideration of developmental timing. Front Hum Neurosci 2010;3:68.

8. Frodl T, Reinhold E, Koutsouleris N, Reiser M, Meisenzahl EM. Interaction of childhood stress with hippocampus and prefrontal cortex volume reduction in major depression. J Psychiatr Res 2010;44:799-807.

9. Samplin E, Ikuta T, Malhotra AK, Szeszko PR, Derosse P. Sex differences in resilience to childhood maltreatment: effects of trauma history on hippocampal volume, general cognition and subclinical psychosis in healthy adults. J Psychiatr Res 2013;47:1174-1179.

10. Sapolsky RM. Stress and plasticity in the limbic system. Neurochem Res 2003;28:1735-1742.

11. Anagnostaras SG, Gale GD, Fanselow MS. Hippocampus and 
contextual fear conditioning: recent controversies and advances. Hippocampus 2001;11:8-17.

12. Maren S, Phan KL, Liberzon I. The contextual brain: implications for fear conditioning, extinction and psychopathology. Nat Rev Neurosci 2013;14:417-428.

13. Pohlack ST, Nees F, Liebscher C, Cacciaglia R, Diener SJ, Ridder $\mathrm{S}$, et al. Hippocampal but not amygdalar volume affects contextual fear conditioning in humans. Hum Brain Mapp 2012;33:478-488.

14. Neueder D, Andreatta M, Pauli P. Contextual fear conditioning and fear generalization in individuals with panic attacks. Front Behav Neurosci 2019;13:152.

15. Woon FL, Sood S, Hedges DW. Hippocampal volume deficits associated with exposure to psychological trauma and posttraumatic stress disorder in adults: a meta-analysis. Prog Neuropsychopharmacol Biol Psychiatry 2010;34:1181-1188.

16. Jackowski AP, de Araújo CM, de Lacerda AL, Mari Jde J, Kaufman J. Neurostructural imaging findings in children with post-traumatic stress disorder: brief review. Psychiatry Clin Neurosci 2009;63:1-8.

17. Utsunomiya H, Takano K, Okazaki M, Mitsudome A. Development of the temporal lobe in infants and children: analysis by MR-based volumetry. AJNR Am J Neuroradiol 1999;20:717-723.

18. Uematsu A, Matsui M, Tanaka C, Takahashi T, Noguchi K, Suzuki M, et al. Developmental trajectories of amygdala and hippocampus from infancy to early adulthood in healthy individuals. PLoS One 2012;7:e46970.

19. Gogtay N, Nugent TF 3rd, Herman DH, Ordonez A, Greenstein D, Hayashi KM, et al. Dynamic mapping of normal human hippocampal development. Hippocampus 2006; 16:664-672.

20. DeMaster D, Pathman T, Lee JK, Ghetti S. Structural development of the hippocampus and episodic memory: developmental differences along the anterior/posterior axis. Cereb Cortex 2014;24:3036-3045.

21. Giovanello KS, Schnyer D, Verfaellie M. Distinct hippocampal regions make unique contributions to relational memory. Hippocampus 2009;19:111-117.

22. Lupien SJ, McEwen BS, Gunnar MR, Heim C. Effects of stress throughout the lifespan on the brain, behaviour and cognition. Nat Rev Neurosci 2009;10:434-445.

23. Vythilingam M, Anderson ER, Goddard A, Woods SW, Staib $\mathrm{LH}$, Charney DS, et al. Temporal lobe volume in panic disorder: a quantitative magnetic resonance imaging study. Psychiatry Res 2000;99:75-82.

24. Dantendorfer K, Prayer D, Kramer J, Amering M, Baischer W, Berger $\mathrm{P}$, et al. High frequency of EEG and MRI brain abnormalities in panic disorder. Psychiatry Res 1996;68:41-53.

25. de Toledo-Morrell L, Dickerson B, Sullivan MP, Spanovic C, Wilson R, Bennett DA. Hemispheric differences in hippocampal volume predict verbal and spatial memory performance in patients with Alzheimer's disease. Hippocampus
2000;10:136-142.

26. Harrison PJ. The hippocampus in schizophrenia: a review of the neuropathological evidence and its pathophysiological implications. Psychopharmacology (Berl) 2004;174:151-162.

27. Woolard AA, Heckers S. Anatomical and functional correlates of human hippocampal volume asymmetry. Psychiatry Res 2012;201:48-53.

28. Levita L, Bois C, Healey A, Smyllie E, Papakonstantinou E, Hartley T, et al. The Behavioural Inhibition System, anxiety and hippocampal volume in a non-clinical population. Biol Mood Anxiety Disord 2014;4:4.

29. Qiu A, Wang L, Younes L, Harms MP, Ratnanather JT, Miller $\mathrm{Ml}$, et al. Neuroanatomical asymmetry patterns in individuals with schizophrenia and their non-psychotic siblings. Neuroimage 2009;47:1221-1229.

30. Zaidel DW, Chiong JK, Merris GJ. Human postnatal developing hippocampus: interhemispheric morphometric comparisons of the dentate gyrus. Soc Neurosci Abstr 1997;23:903.

31. Kerwin RW, Patel S, Meldrum BS, Czudek C, Reynolds GP. Asymmetrical loss of g/utamate receptor subtype in left hippocampus in schizophrenia. Lancet 1988;1:583-584.

32. Oldfield RC. The assessment and analysis of handedness: the Edinburgh inventory. Neuropsychologia 1971;9:97-113.

33. First MB, Spitzer RL, Gibbon M, Williams JB. User's guide for the structured clinical interview for DSM-IV-TR axis I disorders: SCID-I. New York:New York State Psychiatric Institute;2002.

34. Bremner JD, Bolus R, Mayer EA. Psychometric properties of the early trauma inventory-self report. J Nerv Ment Dis 2007; 195:211-218.

35. Jeon JR, Lee EH, Lee SW, Jeong EG, Kim JH, Lee D, et al. The early trauma inventory self report-short form: psychometric properties of the Korean version. Psychiatry Investig 2012;9: 229-235.

36. Lim YJ, Yu BH, Kim JH. Korean Anxiety Sensitivity IndexRevised: its factor structure, reliability, and validity in clinical and nonclinical samples. Depress Anxiety 2007;24:331-341.

37. Taylor S, Cox BJ. An expanded anxiety sensitivity index: evidence for a hierarchic structure in a clinical sample. J Anxiety Disord 1998;12:463-483.

38. Lim YJ, Yu BH, Kim JH. Korean panic disorder severity scale: construct validity by confirmatory factor analysis. Depress Anxiety 2007;24:95-102.

39. Shear MK, Brown TA, Barlow DH, Money R, Sholomskas DE, Woods SW, et al. Multicenter collaborative panic disorder severity scale. Am J Psychiatry 1997;154:1571-1575.

40. Rapee RM, Craske MG, Barlow DH. Assessment instrument for panic disorder that includes fear of sensation-producing activities: the Albany Panic and Phobia Questionnaire. Anxiety 1994;1:114-122.

41. Beck AT, Epstein N, Brown G, Steer RA. An inventory for measuring clinical anxiety: psychometric properties. J Consult Clin Psychol 1988;56:893-897. 
42. Beck AT, Steer RA, Brown GK. BDI-II: Beck depression inventory. San Antonio:Psychological Corp.; 1996. p.490-498.

43. Beck AT, Kovacs M, Weissman A. Assessment of suicidal intention: the scale for suicide ideation. J Consult Clin Psychol 1979; 47:343-352.

44. Brown GK, Beck AT, Steer RA, Grisham JR. Risk factors for suicide in psychiatric outpatients: a 20-year prospective study. J Consult Clin Psychol 2000;68:371-377.

45. Fischl B, Dale AM. Measuring the thickness of the human cerebral cortex from magnetic resonance images. Proc Natl Acad Sci U S A 2000;97:11050-11055.

46. Iglesias JE, Augustinack JC, Nguyen K, Player CM, Player A, Wright $\mathrm{M}$, et al. A computational atlas of the hippocampal formation using ex vivo, ultra-high resolution MRI: application to adaptive segmentation of in vivo MRI. Neuroimage 2015; 115:117-137.

47. Branco DM, Suarez RO, Whalen S, O'Shea JP, Nelson AP, da Costa JC, et al. Functional MRI of memory in the hippocampus: laterality indices may be more meaningful if calculated from whole voxel distributions. Neuroimage 2006;32: 592-602.

48. Lawrie SM, Abukmeil SS. Brain abnormality in schizophrenia. A systematic and quantitative review of volumetric magnetic resonance imaging studies. Br J Psychiatry 1998;172:110120.

49. Csernansky JG, Wang L, Jones D, Rastogi-Cruz D, Posener JA, Heydebrand G, et al. Hippocampal deformities in schizo- phrenia characterized by high dimensional brain mapping. Am J Psychiatry 2002;159:2000-2006.

50. Nogovitsyn N, Muller M, Souza R, Hassel S, Arnott SR, Davis $\mathrm{AD}$, et al. Hippocampal tail volume as a predictive biomarker of antidepressant treatment outcomes in patients with major depressive disorder: a CAN-BIND report. Neuropsychopharmacology 2020;45:283-291.

51. Morris R. Theories of hippocampal function. In: Andersen $P$, Morris R, Amaral D, Bliss T, O'Keefe J, editors. The hippocampus book. New York:Oxford University Press;2007.

52. Cenquizca LA, Swanson LW. Spatial organization of direct hippocampal field CA1 axonal projections to the rest of the cerebral cortex. Brain Res Rev 2007;56:1-26.

53. Fanselow MS, Dong HW. Are the dorsal and ventral hippocampus functionally distinct structures? Neuron 2010;65:7-19.

54. Lee SW, Yoo JH, Kim KW, Kim D, Park H, Choi J, et al. Hippocampal subfields volume reduction in high schoolers with previous verbal abuse experiences. Clin Psychopharmacol Neurosci 2018;16:46-56.

55. Trotman GP, Gianaros PJ, Veldhuijzen van Zanten JJCS, Williams SE, Ginty AT. Increased stressor-evoked cardiovascular reactivity is associated with reduced amygdala and hippocampus volume. Psychophysiology 2019;56:e13277.

56. Klauke B, Deckert J, Reif A, Pauli P, Domschke K. Life events in panic disorder-an update on "candidate stressors". Depress Anxiety 2010;27:716-730. 